\title{
Provider and Staff Morale, Job Satisfaction, and Burnout over a 4-Year Medical Home Intervention
}

\author{
Robert S. Nocon, $\mathrm{MHS}^{1,2,3}$, Paige C. Fairchild, $\mathrm{MPH}^{7}$, Yue Gao, $\mathrm{MPH}^{7}$, \\ Kathryn E. Gunter, MPH, MSW', Sang Mee Lee, PhD², Michael Quinn, PhD', \\ Elbert S. Huang, MD, MPH' , and Marshall H. Chin, $M D, M P H^{7}$
}

\footnotetext{
'Department of Medicine, University of Chicago, Chicago, IL, USA; ${ }^{2}$ Department of Public Health Sciences, University of Chicago, Chicago, IL, USA; ${ }^{3}$ University of Chicago, Chicago, IL, USA.
}

BACKGROUND: The patient-centered medical home $(\mathrm{PCMH})$ is a widely adopted primary care model. However, it is unclear whether changes in provider and staff perceptions of clinic PCMH capability are associated with changes in provider and staff morale, job satisfaction, and burnout in safety net clinics.

OBJECTIVE: To determine how provider and staff PCMH ratings changed under a multi-year PCMH transformation initiative and assess whether changes in provider and staff $\mathrm{PCMH}$ ratings were associated with changes in morale, job satisfaction, and burnout.

DESIGN: Comparison of baseline (2010) and postintervention (2013-2014) surveys.

SETTING: Sixty clinics in five states.

PARTICIPANTS: Five hundred thirty-six (78.2\%) providers and staff at baseline and 589 (78.3\%) post-intervention.

INTERVENTION: Collaborative learning sessions and onsite coaching to implement PCMH over 4 years.

MEASUREMENTS: Provider and staff PCMH ratings on 0 (worst) to 100 (best) scales; percent of providers and staff reporting good or better morale, job satisfaction, and freedom from burnout.

RESULTS: Almost half of safety net clinics improved PCMH capabilities from the perspective of providers (28 out of $59,47 \%$ ) and staff ( 25 out of $59,42 \%$ ). Over the same period, clinics saw a decrease in the percentage of providers reporting high job satisfaction $(-12.3 \%$ points, $p=.009)$ and freedom from burnout $(-10.4 \%$ points, $p=.006)$. Worsened satisfaction was concentrated among clinics that had decreased PCMH rating, with those clinics seeing far fewer providers report high job satisfaction ($38.1 \%$ points, $p<0.001)$

LIMITATIONS: Control clinics were not used. Individuallevel longitudinal survey administration was not feasible. CONCLUSION: If clinics pursue PCMH transformation and providers do not perceive improvement, they may risk

Prior Presentations An earlier version of some content included in this paper was previously presented as a poster presentation at the AcademyHealth Annual Research Meeting (June 2014, San Diego, $C A$ - presented by Paige Fairchild).

Electronic supplementary material The online version of this article (https://doi.org/10.1007/s11606-019-04893-z) contains supplementary material, which is available to authorized users.

Received February 15, 2018

Revised September 20, 2018

Accepted January 24, 2019

Published online March 18, 2019 significantly worsened job satisfaction. Clinics should be aware of this potential risk of PCMH transformation and ensure that providers are aware of PCMH improvements.

KEY WORDS: primary care; safety net; medical home; morale; job satisfaction.

J Gen Intern Med 34(6):952-9

DOI: $10.1007 / \mathrm{s} 11606-019-04893-z$

(c) Society of General Internal Medicine 2019

\section{INTRODUCTION}

Primary care providers and staff commonly experience low morale, poor job satisfaction, and burnout, and attribute these experiences to lack of autonomy, inadequate support from colleagues and leadership, low income, time pressure during office visits, and chaotic work pace. ${ }^{1,2}$ Providers and staff in safety net clinics may experience these issues more acutely, as they are more likely to be affected by resource constraints, personnel shortages, and high demand for care from patients with complex care needs. ${ }^{3-6}$ Morale, job satisfaction, and burnout among safety net providers are increasingly important national health policy concerns, as the Affordable Care Act has led to increased demand for care in safety net clinics while proposals for future reforms to Medicaid may lead to even further resource constraints in the safety net.

Advocates of the patient-centered medical home (PCMH) hope that the model will result in improvements to provider and staff morale and job satisfaction and reductions in burnout. $^{7-9}$ Previous studies in a managed care setting and the US Veterans Health Administration (VA) concluded that PCMH implementation was associated with decreased burnout. ${ }^{10,11}$ However, a second VA study that improved upon adjustment for confounders concluded that burnout increased among VA providers overall, regardless of the level of PCMH implementation. ${ }^{12}$ Another study of PCMH interventions in two states found improvements in job satisfaction in one state and noted that the lack of observed improvement in the other state may have been due to higher baseline levels of satisfaction and a more intensive and stressful PCMH implementation process. ${ }^{13}$

We are aware of only one previous study that assessed changes in job satisfaction, staff morale, and burnout over time among 
non-VA safety net clinics implementing the PCMH. ${ }^{14}$ The study followed a national sample of 296 federally qualified health centers participating in a PCMH improvement intervention and found worsened burnout and satisfaction among providers and staff over a 1-year period. While the study shows that there have been general declines in professional satisfaction over time in safety net clinics pursuing the $\mathrm{PCMH}$, it is also valuable to understand whether the level of perceived PCMH improvement in an intervention is associated with the change in morale, satisfaction, and burnout. We studied this research question in the Safety Net Medical Home Initiative (SNMHI), the first national PCMH demonstration project in the safety net setting, conducted between 2009 and 2013.

\section{METHODS}

We studied 60 of the 65 safety net clinics in the Commonwealth Fund-supported SNMHI. ${ }^{15}$ The goal of the SNMHI was to transform safety net clinics in Colorado, Idaho, Massachusetts, Oregon, and Pennsylvania into high-performing medical homes. Regional coordinating centers provided clinics with collaborative learning sessions and on-site coaching during PCMH implementation. PCMH adoption was structured along eight "change concepts" developed by national technical assistance providers. ${ }^{16}$ Among the original 65 participating clinics, one clinic left the intervention, one clinic merged with another study clinic, and three clinics declined to participate in the post-intervention surveys, leaving 60 clinics for analysis. Among these 60 clinics, one clinic in Colorado had no provider respondents at baseline and was excluded from provider analysis; one clinic in Massachusetts had no staff respondents at baseline and was excluded from the staff analysis. Therefore, 59 clinics were included in the final clinic-level analysis for both the provider and staff models. This study was approved by the University of Chicago Institutional Review Board.

We conducted two mailed, self-administered surveys among providers and clinical staff working at least half time, first between January and June 2010 (baseline) and again between August 2013 and November 2014 (postintervention). For each survey administration, we randomly selected up to 15 individuals per clinic - nine providers and six staff - to receive a survey. Providers included physicians, nurse practitioners, and physician assistants. Clinical staff included behavioral health specialists, educators, medical assistants, dieticians, nurses (licensed practical nurses and registered nurses), psychiatrists, psychologists, and social workers. Each individual surveyed received a one-time incentive of $\$ 10$ included in the initial survey mailing. Among the clinics included in this evaluation, surveys were mailed to 728 providers and clinical staff at baseline and 752 providers and clinical staff post-intervention. Full details on the survey are available in a previous publication. ${ }^{17}$

\section{PCMH Rating}

We assessed PCMH rating, the key independent variable, from the perspective of providers and clinical staff. The survey included 24 questions about the PCMH capabilities of the clinic, organized along five subscales: access to care and communication with patients, communication with other providers, tracking data, care management, and quality improvement. ${ }^{17}$ For providers, the PCMH rating was calculated as the mean of all five subscale scores. For staff, the PCMH rating was calculated based on the mean of four subscales, since the subscale "communication with other providers" was excluded from the staff survey. The PCMH rating score could range from 0 (worst) to 100 (best) for each provider and staff respondent. Provider and staff scores were averaged separately to generate clinic-level PCMH scores. For each clinic, we calculated the change in provider and staff $\mathrm{PCMH}$ rating, as the average postintervention $\mathrm{PCMH}$ rating minus average baseline $\mathrm{PCMH}$ rating for each group.

\section{Provider and Staff Ratings of Morale, Job Satisfaction, and Burnout}

We assessed provider and staff morale, job satisfaction, and burnout, the key dependent variables, with single-question instruments on 5-point Likert-type scales. Staff morale was rated from "poor" to "excellent." Job satisfaction was measured by the level of agreement with the statement "Overall, I am satisfied with my current job". Burnout was measured by response options ranging from "I enjoy my work. I have no symptoms of burnout," to "I feel completely burned out and often wonder if I can go on.". ${ }^{17,} 18$ The scales were collapsed into three individual binary responses. Each of the three outcomes was measured at the individual level and then aggregated into clinic level as percent provider respondents and percent staff respondents reporting good to excellent morale, reporting that they agree or strongly agree with overall job satisfaction, and reporting no symptoms or feelings of burnout.

\section{Covariates}

In our models, we included covariates that have been associated in previous studies with morale, job satisfaction, and burnout: baseline staffing shortages of providers and nurses, change in presence of an electronic medical record (EMR), change in the average number of years since the end of clinical training, and change in work environment score. For providers, models include a covariate for average provider perception of PCMH capability for the clinic at baseline; staff models include the corresponding covariate from staff respondents. Baseline staffing shortages were obtained from a baseline organizational survey ${ }^{19}$ Change in presence of EMR was a three-level variable: no EMR at both time points, presence of an EMR at both time points, and change from no EMR to presence of an EMR from baseline to post-intervention. Work environment was assessed with eight questions on 5-point Likert-type scales. Each response was 
converted to a 0 (worst) to 100 (best) scale and the eight scores were averaged to obtain the a work environment score for each respondent. ${ }^{17}$ For clinic-level analyses, work environment scores were averaged across respondents for each clinic. Average provider- and staff-reported numbers of years since the end of clinical training and ratings for work environment were aggregated for each clinic.

\section{Statistical Analysis}

We generated descriptive statistics for respondents at baseline and post-intervention. We modeled outcomes for providers and clinical staff separately. Exploratory data analysis suggested a nonlinear relationship between change in PCMH change score and levels of morale, satisfaction, and burnout; therefore, we analyzed PCMH score change as a four-level categorical variable: clinics with decreased PCMH score (score change of more than -3 points), clinics with unchanged PCMH score (score change of 3 to +3 points), clinics with moderately increased PCMH score (score change of +3 to +9 points), and clinics with greatly increased PCMH score (score change of more than +9 points). To determine the cut-offs for the four PCMH score change categories, we first examined quartiles of PCMH score change for providers and staff and chose a set of score cut-offs that resulted in categories with roughly even numbers of clinics in each category for both provider and staff analyses. Because provider and staff PCMH scores may differ within the same clinic, the number of clinics in each category differs for provider- and staff-rated PCMH score change.

In unadjusted analyses, we calculated the change in percent of providers reporting good or better morale, high levels of job satisfaction, or no symptoms of burnout for each of the four PCMH score change categories (decrease, no change, moderate increase, and large increase). We used one-way ANOVA to determine whether the change in outcome differed by PCMH change score group. In adjusted analyses, we used multivariate linear regression to model the change in morale, satisfaction, and burnout for each PCMH change score group, while controlling for clinic and provider characteristics. We tested whether the change in outcome for each PCMH score change group is statistically significantly different from zero and report predicted values for change in each outcome by group.

Three clinics had missing values for the years since the end of clinical training and four clinics were missing post-intervention EMR status. We describe our handling of missing data and tests for different approaches to modeling covariates in the Appendix.

All analyses were conducted using SAS, version 9.4 (SAS Institute).

\section{RESULTS}

\section{Provider, Staff, and Clinic Characteristics}

Table 1 describes the characteristics of respondents, and the 60 clinics at baseline and post-intervention. At baseline, we received 291 surveys (78.6\% response rate) from providers and
275 surveys (76.8\% response rate) from clinical staff. Postintervention, we received 305 surveys (75.7\% response rate) from providers and 284 surveys (81.4\% response rate) from clinical staff. At the respondent level, the clinical staff in the post-intervention survey had less clinical experience than clinical staff at baseline (11.3 years vs. 14.8 years, $p=.001$ ). At the clinic level, adoption of electronic medical records increased from $70 \%$ at baseline to $95 \%$ post-intervention $(p=.002)$.

\section{Change in PCMH Ratings, Morale, Job Satisfaction, and Burnout}

The mean change in provider-rated PCMH score across all clinics was a 3.0 point increase (62.1 at baseline to 65.1 postintervention, $p=.046$ ). Perceived improvement was lower among staff, with clinics seeing an average 1.9 point improvement in staff-rated PCMH score (66.9 at baseline to 68.8 postintervention, $p=.051$ ) (Table 2).

While average change across all clinics was relatively small, individual clinics' experiences varied notably. Fourteen clinics (24\%) saw a decrease of more than three points in average provider rating of PCMH capability, 17 (29\%) showed no change, 13 (22\%) showed a moderate increase of three to nine points, and $15(25 \%)$ showed a large increase of over nine points in provider-rated PCMH score. From the perspectives of staff respondents, 13 clinics $(22 \%)$ had a decrease in average staff rating, $21(36 \%)$ showed no change, $15(25 \%)$ showed a moderate increase, and $10(17 \%)$ showed a large increase in staff-rated PCMH score.

Over the course of the intervention, there was a significant decrease in the percent of providers reporting high levels of job satisfaction (12.3 percentage point decrease, from $82.2 \%$ at baseline to $69.8 \%$ post-intervention, $p=.009)$ and freedom from burnout (10.4 percentage point decrease, from $63.9 \%$ at baseline to $53.5 \%$ post-intervention, $p=.006$ ).

ASSOCIATION OF CHANGE IN PCMH RATING WITH MORALE, JOB SATISFACTION, AND BURNOUT.

In unadjusted analyses (Table 3), for clinics with decreased provider-rated PCMH scores, the percent of providers reporting good or better morale decreased by 27.9 percentage points $(95 \%$ confidence interval $[95 \% \mathrm{CI}]-50.5,-5.4)$, the percent of providers reporting high levels of job satisfaction decreased by 48.9 points $(95 \% \mathrm{CI}-64.2,-33.6)$, and the percent of providers reporting freedom from burnout decreased by 22.2 points $(95 \%$ CI - 39.4, -4.9). In clinics with moderate increases in providerrated PCMH scores, the percent of providers reporting freedom from burnout decreased by 15.8 points $(95 \%$ CI $-33.0,-1.5)$. In clinics with decreased staff-rated PCMH scores, the percent of staff reporting good or better morale decreased by 27.4 points $(95 \%$ CI $-45.8,-8.9)$. Analysis of variance showed that changes in provider job satisfaction $(p<0.001)$ and staff morale $(p<0.009)$ were statistically significantly different between PCMH change score groups.

In adjusted analyses (Table 4) that control for baseline PCMH rating, baseline provider shortage, nursing shortage, electronic 
Table 1 Characteristics of Providers, Staff, and Clinics

\begin{tabular}{|c|c|c|c|c|c|c|}
\hline & \multicolumn{3}{|l|}{ Providers } & \multicolumn{3}{|l|}{ Staff } \\
\hline & Baseline & Post-intervention & $p$ value & Baseline & Post-intervention & $p$ value \\
\hline No. of respondents (response rate, \%) & $291(78.6)$ & $305(75.7)$ & & $275(76.8)$ & $284(81.4)$ & \\
\hline Female $(\%)$ & 65.2 & 66.0 & 0.83 & 91.9 & 91.1 & 0.74 \\
\hline Race/ethnicity (\%) & & & 0.96 & & & 0.51 \\
\hline White & 83.0 & 81.5 & & 63.0 & 56.7 & \\
\hline Black & 3.8 & 4.3 & & 5.6 & 8.5 & \\
\hline Hispanic or Latino & 5.5 & 7.0 & & 25.6 & 27.3 & \\
\hline Other & 6.2 & 6.0 & & 4.4 & 5.3 & \\
\hline Multi-race & 1.4 & 1.3 & & 1.5 & 2.1 & \\
\hline Provider or staff type $(\%)$ & & & 0.18 & & & 0.32 \\
\hline Physician & 65.0 & 59.7 & & $\mathrm{~N} / \mathrm{A}$ & $\mathrm{N} / \mathrm{A}$ & \\
\hline Nurse practitioner or physician assistant & 35.1 & 40.3 & & $\mathrm{~N} / \mathrm{A}$ & $\mathrm{N} / \mathrm{A}$ & \\
\hline Registered nurse & $\mathrm{N} / \mathrm{A}$ & $\mathrm{N} / \mathrm{A}$ & & 29.5 & 25.4 & \\
\hline Licensed practical nurse or medical assistant & $\mathrm{N} / \mathrm{A}$ & $\mathrm{N} / \mathrm{A}$ & & 46.6 & 52.8 & \\
\hline Other & $\mathrm{N} / \mathrm{A}$ & $\mathrm{N} / \mathrm{A}$ & & 24.0 & 21.8 & \\
\hline Years since end of clinical training, mean (SD) & $11.8(9.4)$ & $12.2(10.0)$ & 0.67 & $14.8(12.5)$ & $11.3(11.4)$ & 0.001 \\
\hline Years working at this clinic, mean (SD) & $6.7(6.2)$ & $7.0(6.6)$ & 0.65 & $5.6(5.8)$ & $5.2(5.2)$ & 0.42 \\
\hline Primary patient population $(\%)$ & & & 0.24 & & & 0.19 \\
\hline Children $<18$ years & 7.3 & 8.9 & & 3.4 & 5.1 & \\
\hline Adults $\geq 18$ years & 22.0 & 26.8 & & 25.0 & 30.4 & \\
\hline \multirow[t]{3}{*}{ Children and adults } & 70.7 & 64.2 & & 71.6 & 64.5 & \\
\hline & \multicolumn{3}{|c|}{ Clinics $(n=60)$} & & & \\
\hline & Baseline & Post-intervention & $p$ value & & & \\
\hline \multicolumn{7}{|l|}{ State of clinic $(\%)$} \\
\hline Colorado & 22 & $\mathrm{~N} / \mathrm{A}$ & & & & \\
\hline Idaho & 20 & $\mathrm{~N} / \mathrm{A}$ & & & & \\
\hline Massachusetts & 22 & $\mathrm{~N} / \mathrm{A}$ & & & & \\
\hline Oregon & 23 & $\mathrm{~N} / \mathrm{A}$ & & & & \\
\hline Pennsylvania & 13 & $\mathrm{~N} / \mathrm{A}$ & & & & \\
\hline \multicolumn{7}{|l|}{ Location $(\%)$} \\
\hline City & 50 & $\mathrm{~N} / \mathrm{A}$ & & & & \\
\hline Suburban & 8 & $\mathrm{~N} / \mathrm{A}$ & & & & \\
\hline Small town & 15 & $\mathrm{~N} / \mathrm{A}$ & & & & \\
\hline Rural & 18 & $\mathrm{~N} / \mathrm{A}$ & & & & \\
\hline Frontier & 8 & $\mathrm{~N} / \mathrm{A}$ & & & & \\
\hline Executive director reported EMR adoption (\%) & 70 & 95 & 0.002 & & & \\
\hline Total number of provider full-time equivalents $(\%)$ & & & 0.12 & & & \\
\hline$<4$ & 35 & 20 & & & & \\
\hline $4-8$ & 27 & 36 & & & & \\
\hline$>8$ & 38 & 45 & & & & \\
\hline Provider shortage $(\%)$ & 48 & 59 & 0.22 & & & \\
\hline Nursing shortage $(\%)$ & 23 & 25 & 1.00 & & & \\
\hline
\end{tabular}

For provider characteristics, baseline to post-intervention differences were tested with paired t tests for continuous variables and chi-square tests for categorical variables

For clinic characteristics, baseline and post-intervention differences were tested with McNemar's test

medical record status, and change in average provider/staffreported number of years since the end of clinical training, clinics with decreased provider-rated PCMH score saw a 38.1 percentage point decline in the percent of providers reporting satisfaction with their job (95\% CI - 56.7, - 19.5) and a 21.5 percentage point decline in the percent of providers reporting freedom from burnout (95\% CI - 42.9, - 0.04). We did not observe statistically significant changes in morale, satisfaction, or burnout in any of the other three clinic groups (those with no change, moderate increase, or large increase in PCMH score). Even among clinics with a large increase in PCMH score, estimates of change in morale, satisfaction, and burnout were positive, but smaller in magnitude and not statistically significant.

\section{DISCUSSION}

From the perspective of providers and staff, the SNMHI was associated with small improvements in PCMH capability overall, though results varied across clinics. Almost half of clinics saw small or large improvements in their PCMH ratings and almost one-fourth saw a decline. A decline in provider PCMH rating was associated with large decreases in the rate of provider job satisfaction. In contrast, even large increases in PCMH rating were not associated with statistically significant improvements in morale, satisfaction, or burnout.

Our finding of small overall improvement in provider- and staff-perceived PCMH characteristics illustrates a critical challenge of large-scale, facilitated PCMH improvement work. Past studies of the SNMHI have shown how the intervention improved perception of PCMH characteristics among the teams working to implement the model in the clinic. ${ }^{15}$ It may be more difficult - though still possible - to improve provider and staff perceptions of PCMH characteristics across a range of providers and staff that may have less direct participation in the implementation process. 
The large overall decrease in provider job satisfaction and increase in burnout that we observed in our study is also notable and consistent with the only previous longitudinal study of these outcomes in a non-VA safety net PCMH implementation. ${ }^{14}$ Our study did not have a control group of clinics that would allow us to identify secular trends in job satisfaction and burnout that would have occurred outside the context of PCMH transformation. It may be that these clinics would have seen further worsening of satisfaction and burnout in the absence of the intervention.

Worsened satisfaction and burnout over the course of this intervention may also be explained by considering the categories of clinic-level change in PCMH rating. Roughly onefourth of the clinics saw decreases in provider- or staffperceived PCMH score, and those clinics experienced extremely large decreases in provider job satisfaction. For these clinics, providers not only saw the PCMH initiative as unsuccessful, but they perceived that the initiative made things worse. This perception is compounded with the fact that PCMH transformation initiatives are generally demanding organizational changes. The corresponding change in provider job satisfaction for this group of clinics was more than four times worse than clinics where providers simply perceived a lack of improvement in PCMH capability. This pattern may illustrate a particularly significant risk of PCMH implementation efforts that those implementing the model should work to proactively address. In past qualitative assessments of the SNMHI, individuals involved in PCMH implementation highlighted the importance of regular reporting of PCMH progress and success to providers and staff throughout the clinic, as well as the need to ensure that providers and staff buy-in to the changes pursued. ${ }^{9}$ If such efforts can lessen the likelihood that providers perceive a worsening of PCMH capability over the course of a transformation effort, they may be an important component of avoiding significantly worsened job satisfaction.

We found consistently positive (though non-significant) changes in all outcomes for clinics that had large increases in PCMH rating. Future studies should examine whether large improvements in PCMH capability could drive even modest improvements in morale, satisfaction, and burnout in safety net settings. This would be particularly notable in light of broader secular declines in those outcomes.

Our study has limitations. First, our study does not establish a causal relationship - changes in job satisfaction may have occurred first and caused lower perceptions of PCMH capabilities. Despite this limitation, we note that this is the first study to assess this relationship in a non-VA safety net setting, and the first to note the particularly large decline in provider job satisfaction associated with decreased provider PCMH rating. Second, worsened provider job satisfaction may have been attributable to factors that we did not measure in this study. Change fatigue or a lack of readiness to change may have independently inhibited clinics' abilities to both adopt the PCMH model and maintain job satisfaction or limit burnout. ${ }^{20}$ 


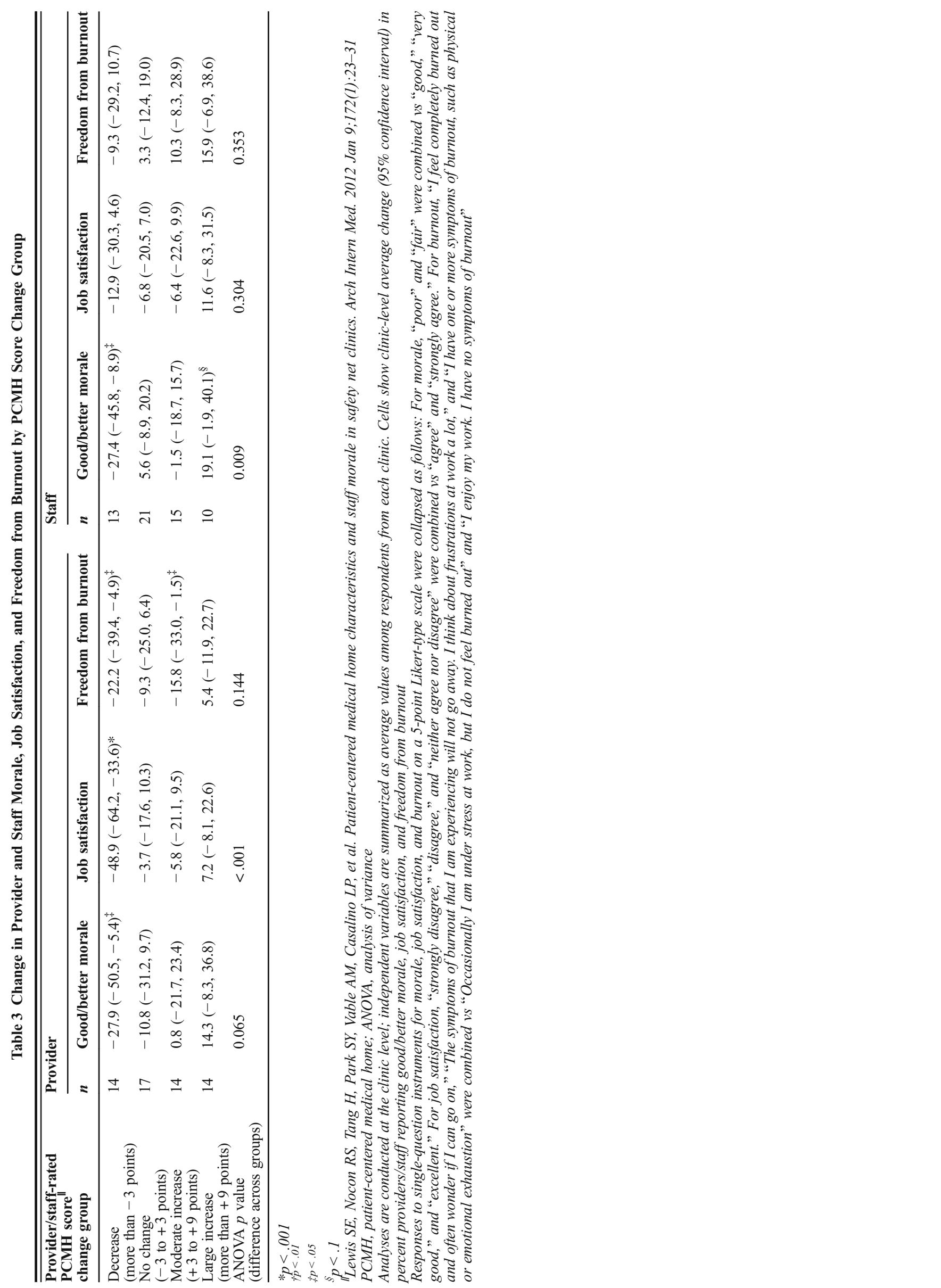




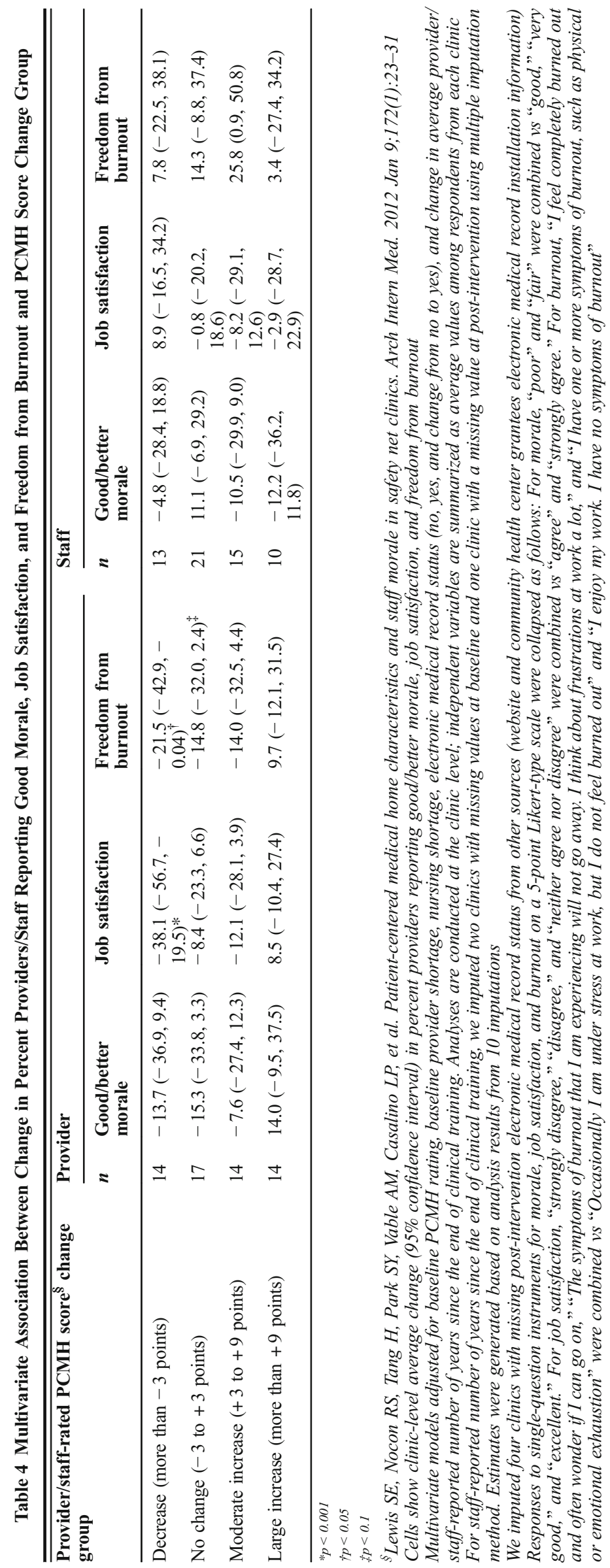


While we include adoption of an EMR as a covariate in the analyses, clinics may also experience replacements or significant changes to their EMRs that may influence PCMH capability and job satisfaction. Third, since we did not administer the surveys to the same providers and staff at both baseline and post-intervention, we cannot measure the change in individual perceptions of PCMH characteristics or morale, job satisfaction, and burnout. Fourth, these results cannot be generalized to all safety net clinics because clinics participating in the SNMHI were not randomly sampled. Finally, our surveys were administered to randomly selected providers and staff in each clinic and we do not know whether those individuals were actively involved in PCMH implementation activities.

Although on average, provider and staff perceptions of PCMH characteristics improved slightly from baseline, provider job satisfaction and freedom from burnout worsened and the effects were concentrated among clinics that had decreased perceptions of PCMH capability. Policymakers and healthcare industry leaders who advocate for the implementation of patient-centered medical homes should keep in mind the challenge of demonstrating PCMH progress throughout their provider population and the significant risks to satisfaction and burnout that may arise when providers do not perceive that progress.

Corresponding Author: Robert S. Nocon, MHS; University of Chicago, 5841 S. Maryland Ave. MC2007, Chicago, IL 60637, USA (e-mail: rnocon@bsd.uchicago.edu).

Funders Financial support for the study was provided by The Commonwealth Fund. Dr. Chin was supported by the National Institute of Diabetes and Digestive and Kidney Diseases (K24 DK071933 and P30 DK092949). Mr. Nocon was supported by an Agency for Healthcare Research and Quality training grant (T32 HSOOOO84).

\section{Compliance with Ethical Standards:}

This study was approved by the University of Chicago Institutional Review Board.

Conflict of Interest: The authors declare that they do not have a conflict of interest.

Publisher's Note: Springer Nature remains neutral with regard to jurisdictional claims in published maps and institutional affiliations.

\section{REFERENCES}

1. Linzer M, Manwell LB, Williams ES, Bobula JA, Brown RL, Varkey AB, et al. Working conditions in primary care: physician reactions and care quality. Ann Intern Med. 2009;151(1):28-36, W6-9.
2. Scheurer D, McKean S, Miller J, Wetterneck T. U.S. physician satisfaction: a systematic review. J Hosp Med. 2009;4(9):560-8.

3. Rosenblatt RA, Andrilla CHA, Curtin T, Hart LG. Shortages of medical personnel at community health centers: implications for planned expansion. J Am Med Assoc. 2006;295(9):1042-1049.

4. Hayashi AS, Selia E, McDonnell $\mathbf{K}$. Stress and provider retention in underserved communities. J Health Care Poor Underserved. 2009;20(3):597-604.

5. Cole AM, Chen FM, Ford PA, Phillips WR, Stevens NG. Rewards and challenges of community health center practice. J Prim Care Community Health. 2014;5(2):148-51.

6. Curlin FA, Serrano KD, Baker MG, Carricaburu SL, Smucker DR, Chin MH. Following the call: how providers make sense of their decisions to work in faith-based and secular urban community health centers. J Health Care Poor Underserved. 2006;17(4):944-57.

7. American Academy of Family Physicians, American Academy of Pediatrics, American College of Physicians, American Osteopathic Association. Joint Principles of the Patient-Centered Medical Home. Patient Centered Primary Care Collaborative; 2007. Available at: http://www.pcpcc.org/ about/medical-home. Accessed January 10, 2019.

8. Friedberg $\mathbf{M W}$. The Potential Impact of the Medical Home on Job Satisfaction in Primary Care. Arch Intern Med. 2012;172(1):31-2.

9. Guinn M, Gunter $\mathbf{K}$, Nocon $\mathbf{R}$, Lewis $\mathbf{S}$, Vable $\mathbf{A}$, Tang $\mathbf{H}$, et al. Undergoing transformation to the patient centered medical home in safety net health centers: perspectives from the front lines. Ethn Dis. 2013;23:356-62.

10. Reid RJ, Coleman K, Johnson EA, Fishman PA, Hsu C, Soman MP, et al. The Group Health medical home at year two: cost savings, higher patient satisfaction, and less burnout for providers. Health Aff (Millwood). 2010;29(5):835-43.

11. Nelson KM, Helfrich C, Sun H, Hebert PL, Liu C-F, Dolan E, et al. Implementation of the patient-centered medical home in the Veterans Health Administration: associations with patient satisfaction, quality of care, staff burnout, and hospital and emergency department use. JAMA Intern Med. 2014;174(8):1350-8.

12. Simonetti JA, Sylling PW, Nelson K, Taylor L, Mohr DC, Curtis I, et al. Patient-Centered Medical Home Implementation and Burnout Among VA Primary Care Employees. J Ambulatory Care Manage. 2017;40(2):15866.

13. Alidina S, Rosenthal MB, Schneider EC, Singer SJ, Friedberg MW. Practice environments and job satisfaction in patient-centered medical homes. Ann Fam Med. 2014;12(4):331-7.

14. Friedberg MW, Reid RO, Timbie JW, Setodji C, Kofner A, Weidmer B, et al. Federally Qualified Health Center Clinicians And Staff Increasingly Dissatisfied With Workplace Conditions. Health Aff Proj Hope. 2017 36(8): 1469-75.

15. Sugarman JR, Phillips KE, Wagner EH, Coleman K, Abrams MK. The safety net medical home initiative: transforming care for vulnerable populations. Med Care. 2014;52:S1-S10.

16. Safety Net Medical Home Initiative Web Site. The change concepts for practice transformation: overview. Available at: http://www. safetynetmedicalhome.org/sites/default/files/Change-Concepts-Overview.pdf. Accessed January 10, 2019.

17. Lewis SE, Nocon RS, Tang H, Park SY, Vable AM, Casalino LP, et al. Patient-centered medical home characteristics and staff morale in safety net clinics. Arch Intern Med. 2012;172(1):23-31.

18. Rohland BM, Kruse GR, Rohrer JE. Validation of a single-item measure of burnout against the Maslach Burnout Inventory among physicians. Stress Health. 2004;20(2):75-9.

19. Birnberg JM, Drum ML, Huang ES, Casalino LP, Lewis SE, Vable AM, et al. Development of a safety net medical home scale for clinics. J Gen Intern Med. 2011;26(12):1418-25.

20. Nutting PA, Miller WL, Crabtree BF, Jaen CR, Stewart EE, Stange KC. Initial Lessons From the First National Demonstration Project on Practice Transformation to a Patient-Centered Medical Home. Ann Fam Med. 2009;7(3):254-60. 